Research Paper

\title{
Comparison of the Effects of BMSC-derived Schwann Cells and Autologous Schwann Cells on Remyelination Using a Rat Sciatic Nerve Defect Model
}

\author{
Bo Hou ${ }^{1^{*}}$, Zhuopeng Ye ${ }^{1^{*}}$, Wanqing $\mathrm{Ji}^{2^{*}}$, Meiqin Cai ${ }^{1}$, Cong Ling ${ }^{1}$, Chuan Chen ${ }^{1}$, Ying Guo ${ }^{1 凶}$ \\ 1. Department of Neurosurgery, The Third Affiliated Hospital, Sun Yat-sen University, Guangzhou, Guangdong Province, 510630, China. \\ 2. Department of Obstetrics, Guangzhou Women and Children's Medical Center, Guangzhou Medical University, Guangzhou, Guangdong Province, 510623, \\ China. \\ *Equal contributors: Bo Hou, Zhuopeng Ye, Wanqing Ji.
}

$\square$ Corresponding author: Ying Guo, Department of Neurosurgery, Third Affiliated Hospital, Sun Yat-sen University, 600 Tian he Road, Guangzhou, China, 510630. Phone number: 86-018922102633; Fax number: 86-20-8525 2170; E-mail: publicsjwk@163.com

(c) Ivyspring International Publisher. This is an open access article distributed under the terms of the Creative Commons Attribution (CC BY-NC) license (https://creativecommons.org/licenses/by-nc/4.0/). See http://ivyspring.com/terms for full terms and conditions.

Received: 2018.04.19; Accepted: 2018.09.08; Published: 2018.10.31

\begin{abstract}
Schwann cells (SCs) are primarily responsible for the formation of myelin sheaths, yet bone marrow mesenchymal stem cell (BMSC)-derived SCs are often used to replace autologous SCs and assist with the repair of peripheral nerve myelin sheaths. In this study, the effects of the two cell types on remyelination were compared during the repair of peripheral nerves. Methods: An acellular nerve scaffold was prepared using the extraction technique. Rat BMSCs and autologous SCs were extracted. BMSCs were induced to differentiate into BMSC-derived SCs (B-dSCs) in vitro. Seed cells (BMSCs, B-dSCs, and autologous SCs) were cocultured with nerve scaffolds ( $\mathrm{Sca}$ ) in vitro. Rats with severed sciatic nerves were used as the animal model. A composite scaffold was used to bridge the broken ends. After surgery, electrophysiology, cell tracking analyses (EdU labeling), immunofluorescence staining (myelin basic protein (MBP)), toluidine blue staining, and transmission electron microscopy were conducted to compare remyelination between the various groups and to evaluate the effects of the seed cells on myelination. One week after transplantation, only a small number of B-dSCs expressed MBP, which was far less than the proportion of MBP-expressing autologous SCs $(P<0.01)$ but was higher than the proportion of BMSCs expressing MBP $(P<0.05)$. Four weeks after surgery, the electrophysiology results (latency time, conductive velocity and amplitude) and various quantitative indicators of remyelination (thickness, distribution, and the number of myelinated fibers) showed that the Sca+B-dSC group was inferior to the Sca+autologous $S C$ group $(P<0.05)$ but was superior to the Sca+BMSC group $(P<0.05)$. Conclusions: Within 4 weeks after surgery, the use of an acellular nerve scaffold combined with B-dSCs promotes remyelination to a certain extent, but the effect is significantly less than that of the scaffold combined with autologous SCs.
\end{abstract}

Key words: Schwann cells; remyelination; bone marrow mesenchymal stem cell; nerve regeneration; cell tracking

\section{Introduction}

Two prerequisites for functional recovery after peripheral nervous system (PNS) injury have been identified: axonal regeneration and remyelination [1-2]. After PNS injury, Wallerian degeneration occurs in the distal nerve, and the locally proliferating Schwann cells (SCs) subsequently remyelinate and modify the regenerated axon[3]. The myelin sheath protects the axons and promotes the rapid and effective transmission of nerve impulses. Eventual remyelination of the regenerated axon is a necessary step for the effective transmission of nerve signals and functional recovery. 
SCs play the most important roles in axon remyelination, and their functions are complex and diverse[4]. SCs have recently been shown to exist in three states[5,6]. In the normal PNS, SCs play a role in forming (myelin states) and maintaining the integrity of the myelin sheath (nonmyelin states, also called Remak cells)[7]. After PNS injury, SCs in the above two states are immediately activated and dedifferentiated into repair Schwann cells[8], which nourish severed axons to promote axon regeneration. These cells act as scavengers to remove the disintegrated myelin debris, and more importantly, they form unique "Büngner bands" to guide the extension of the regenerating axon and establish connections with target sites. When axon regeneration is complete, repair SCs once again shift to a myelinating state, and Remak cells wrap the regenerated axon to complete the remyelination process[9]. For PNS lesions with a longer separation distance (a distance greater than $2 \mathrm{~cm}$ )[10], the proximal regenerated axon has difficulty crossing the lesion to reconnect with the distal axon structure because the injured area lacks the support of SCs and a neural basal lamina scaffold. Because the injured area lacks nerve innervation for a long period of time, the distal axon gradually disintegrates[11-13]. Therefore, SC transplantation into an injury lesion is an effective neural repair strategy[14]. However, many limitations in obtaining and expanding autologous SCs in vitro have been identified. Autologous SCs are not suitable for use as ideal seed cells. Recently, many research groups have turned their attention to the stem cell field to conduct in-depth research and have achieved some promising results[15].

Bone marrow mesenchymal stem cells (BMSCs) are widely used in the field of nerve regeneration. BMSCs have great application advantages in neural differentiation, neuroprotection, and neurotrophy. In particular, BMSCs have recently been successfully and directionally induced to differentiate into BMSC-derived SCs (B-dSCs)[16,17], which not only express PNS glial lineage markers (i.e., S-100, O4, and glial fibrillary acidic protein (GFAP)) but also wrap the axon in a myelin sheath when cocultured with neurons[18]. Moreover, animal experiments have confirmed that these cells play certain roles in promoting neural regeneration in vivo in place of autologous SCs[19,20]. B-dSCs display a similar efficacy to autologous SCs in many respects[21], and we are interested in the extent to which B-dSCs substitute for autologous SCs in vivo. In this project, acellular nerve scaffolds were used as cell transplantation vectors, and rats with a severed sciatic nerve were used as a model to investigate whether these two seed cell populations exerted the same effects on promoting remyelination.

\section{Materials and Methods}

\section{Animals}

Animals: Neonatal Sprague-Dawley (SD) rats aged between 3 and 5 days were used for BMSC isolation, and $88 \mathrm{SD}$ rats (either sex, body weights 180-200 g) were used to prepare the animal models and for autologous SC isolation. All experimental animals were housed under standard conditions, and all protocols were approved by the Experimental Animal Administration and Ethics Committee of Sun Yat-Sen University.

\section{Preparation and detection of the scaffold}

\section{Scaffold preparation}

A $4.5-\mathrm{cm}$ section of the sciatic nerve trunk was removed from an SD rat (used for other experiments) as the raw material for the preparation of the scaffold and was treated as follows: 1 . rinsed with $\mathrm{dd}_{2} \mathrm{O}$ for 2 $\mathrm{h}, 2$. mechanical shaking in $125 \mathrm{mM}$ sulfobetaine- 10 (SB-10) in $\mathrm{ddH}_{2} \mathrm{O}$ for $12 \mathrm{~h}, 3$. rinsed with PBS 3 times ( $5 \mathrm{~min} /$ rinse), 4 . mechanical shaking in $0.14 \%$ Triton X-200 and $0.6 \mathrm{mM}$ sulfobetaine-16 (SB-16) for $24 \mathrm{~h}$, and 5. rinsed with PBS 3 times (5 min/rinse). Steps 2-3 were repeated, and then the material was treated with $4 \%$ sodium deoxycholate for $24 \mathrm{~h}$. Next, $0.1 \%$ (v/v) peracetic acid (Adamas-Beta, Switzerland) diluted in $\mathrm{ddH}_{2} \mathrm{O}$ was used to sterilize the material for $3 \mathrm{~h}$, followed by 3 washes in sterile PBS (10 min/rinse). The scaffold was stored in sterile PBS at $4^{\circ} \mathrm{C}$ until subsequent experiments. All procedures described above were performed at room temperature with shaking on a shaker (150 rpm/min). All chemicals were purchased from Sigma (St. Louis, MO, USA), unless indicated otherwise.

\section{Determination of the structure and composition of the scaffold}

Nerve grafts subjected to the decellularization treatment were embedded in Tissue-Tek ${ }^{\circledR}$ O.C.T. Compound (Sakura Finetek, USA). Frozen longitudinal and transverse sections were cut at a thickness of $5 \mu \mathrm{m}$ using a freezing microtome (Leica, USA) and placed on poly-L-lysine-coated slides.

\section{Composition}

Immunostaining was performed to analyze the scaffold composition. Sections were incubated with a primary antibody (anti-laminin (LN); 1:1000, Sigma-Aldrich, St. Louis, MO, USA, or antifibronectin (FN); 1:400, Abcam, Cambridge, MA, USA) in a humidified chamber at $4^{\circ} \mathrm{C}$ overnight. 
Sections were warmed to room temperature, incubated with a secondary antibody for $1 \mathrm{~h}$ in a humidified chamber at $37^{\circ} \mathrm{C}$, and washed 3 times with 0.1 M PBS ( $\mathrm{pH}$ 7.4) on a shaker (100 r/min). 4',6-Diamidino-2-phenylindole (DAPI; Sigma-Aldrich, St. Louis, MO, USA) was used for counterstaining, and the images were visualized using an Eclipse 80i (Nikon, Japan).

\section{Structure}

The sections prepared as described above were stained with hematoxylin and eosin (H\&E) to confirm that the nuclei had been removed. The transmission electron microscopy detection method is described in detail below.

\section{Preparation of seed cells}

\section{Extraction and culture of BMSCs}

BMSCs were harvested from the bone marrow of neonatal rat femurs. This procedure is briefly described below. Neonatal rat femurs were isolated under aseptic conditions and rinsed three times with PBS. The bone cavity was repeatedly rinsed in the longitudinal direction with a-Minimum Essential Medium (a-MEM; Gibco, USA) containing 10\% fetal bovine serum (FBS; Gibco, USA) and penicillin/ streptomycin $(50 \mathrm{U} / \mathrm{ml}$ and $50 \mathrm{mg} / \mathrm{ml}$, respectively) 15-20 times. The rinse solution was collected, filtered through a 200-mesh sieve, and cultured under standard conditions $\left(37^{\circ} \mathrm{C}\right)$. Twelve hours later, the medium was replaced with fresh medium (used above) to remove unattached cells. Then, the medium was replaced once every 3-4 days, and P3 cells were used for flow cytometry-based identification.

\section{Flow cytometry-based identification of BMSCs}

The concentration of the P3 BMSC suspension was adjusted to $5 \times 10^{6}$ cells $/ \mathrm{ml}$, and the cells were fixed with $4 \%$ neutral paraformaldehyde for $30 \mathrm{~min}$. Each EP tube contained $100 \mu \mathrm{l}$ (approximately 5.0x10 cells) of the suspension. The following antibodies were added to the tubes: CD90-fluorescein isothiocyanate (FITC) as a stem cell marker; CD29-phycoerythrin (PE) and CD44-PE (Serotec Oxford, UK) as mesenchymal lineage markers; and CD11b-PE, CD45-PE, CD106, and CD34-PE (Santa Cruz Biotechnology) as hematopoietic lineage markers. The tube contents were mixed well and incubated at $4^{\circ} \mathrm{C}$ in the dark for $30 \mathrm{~min}$. Then, a BD FACSCalibur flow cytometer was used to analyze the cells. All antibodies used in the flow cytometry analyses were purchased from BioLegend, unless indicated otherwise.
Directionally induced differentiation of BMSCs into B-dSCs

The induction method [18] is briefly described below. P3 generation BMSCs were collected, and the cell concentration was adjusted to $1 \times 10^{5}$ cells $/ \mathrm{ml}$ with mixed induction medium containing 1:1 (v/v) Dulbecco's Modified Eagle's Medium (DMEM)/F12 and Neurobasal medium (Invitrogen) supplemented with basic fibroblast growth factor (bFGF; $40 \mathrm{ng} / \mathrm{ml}$; Peprotech), epidermal growth factor $(20 \mathrm{ng} / \mathrm{ml})$ (Peprotech), and B27 (2\%, v/v, Invitrogen). Then, the cells were seeded onto ultralow attachment culture plates (Corning). Three weeks later, neurospheres were collected and seeded into poly-lysine-coated flasks. After the cells had attached to the wall, the medium was changed to a mixed medium composed of a-MEM (Gibco) supplemented with 10\% FBS, forskolin $(5 \mathrm{mM})$, platelet-derived growth factor (PDGF)-AA (5 ng/ml), bFGF $(10 \mathrm{ng} / \mathrm{ml})$, and $\beta$-heregulin ( $200 \mathrm{ng} / \mathrm{ml})$. Cells were cultured for two additional weeks before the detection of the S-100, O4, and GFAP proteins. These B-dSCs were further cultured in basal medium (DMEM/F12 supplemented with $10 \% \mathrm{FBS}$ ).

\section{Isolation and identification of autologous Schwann cells}

One-half centimeter of the unilateral rat radial nerve was transected (the animal was sutured for the subsequent experiments) and digested with $0.05 \%$ collagenase. The digested mixture was repeatedly pipetted to collect the cell suspension, which was culture in DMEM-F12 containing 10\% fetal bovine serum (in a $37^{\circ} \mathrm{C}$ humidified incubator with $10 \%$ CO2). The mitotic inhibitors cytosine arabinoside (Ara-c) and G-418 were used to remove fibroblasts, and bFGF was used to promote the growth of Schwann cells. After the cells were confluent, the rapid digestion method with a low concentration of trypsin was used to collect the cells. The P3 generation of the cells was stained with the Schwann cell-specific antibody S-100 (1:800, Sigma, St. Louis, MO, USA), O4 (1:1000, Santa Cruz Biotechnology, USA), and GFAP (1:400, Sigma, St. Louis, MO, USA).

\section{Cell labeling}

EdU (5-ethynyl-2'-deoxyuridine) is a DNA thymine base pair analog that is incorporated into DNA when the cells undergo mitosis[22]. Therefore, EdU has been used to evaluate cell proliferation or to replace the traditional BrdU labeling method for cell tracking. The labeling method is described below. A total of $1 \times 10^{6}$ cells were collected and seeded into a culture flask $\left(25 \mathrm{~cm}^{2}\right)$. After all cells had adhered to the flask wall, the Click-iT ${ }^{\mathrm{TM}}$ EdU (Molecular Probes; 
Invitrogen) solvent was diluted with the culture medium to a working concentration of $20 \mu \mathrm{M}$, added to the culture flask, and incubated for $24 \mathrm{~h}$. The medium was replaced with fresh medium. When the cells were traced, $4 \%$ paraformaldehyde was used to fix the specimen for $15 \mathrm{~min}$; then, the cells were stained overnight with the detection medium (Alexa Fluor 594), and the cells were observed under a fluorescence microscope (Eclipse 80i, Nikon). The results were analyzed using Image-Pro Plus 6.0 software (Media Cybernetics, Inc., USA).

\section{Implantation and complex formation}

The cell complex formation method is briefly described below. The acellular nerve scaffold (approximately 2-cm long) prepared as described above was collected. A microsyringe was used to withdraw a 100- $\mu$ l cell suspension (approximately $1 \times 10^{6}$ cells), which was slowly injected into the scaffold. The surface of the scaffold was rinsed 3 times with PBS (3 min/rinse). Then, the nerve scaffold complexed with cells was placed into culture medium (DMEM/F12 supplemented with 10\% FBS) and cultured for 7 days. Frozen sections were prepared for immunostaining (S-100), scanning electron microscopy (SEM), or animal experiments. For the SEM analysis, the specimen was fixed with glutaraldehyde for $2 \mathrm{~h}$ and postfixed with a $4 \%$ osmic acid solution for $2 \mathrm{~h}$. After dehydration in a gradient of alcohol solutions, the specimen was soaked in isoamyl acetate for $15 \mathrm{~min}$ to replace the alcohol content in the specimen. Critical point drying of the specimen was performed for $4 \mathrm{~h}$ (Hitachi, Tokyo, Japan). The dried specimens were coated with gold and observed using a Quanta 200 SEM (FEI, USA).

\section{Surgical methods}

Eighty-eight SD rats were randomly divided into the 9 groups described in Table 1 according to the administration of different treatments. Sodium pentobarbital $(30 \mathrm{mg} / \mathrm{ml}, 0.4 \mathrm{ml} / 100 \mathrm{~g})$ was injected intraperitoneally, and the right sciatic nerve was exposed. The trunk of the sciatic nerve was completely severed at $0.5 \mathrm{~cm}$ proximal to the branch of the tibial nerve and peroneal nerve to cause an approximately $1.5-\mathrm{cm}$-long nerve defect. For the Sca+B-dSC, Sca+BMSC, Sca+autologous SC and Sca groups, the $1.5-\mathrm{cm}$ nerve scaffold complexed with cells was used to bridge the two ends of the defect region using the adventitia suture method. For the B-dSC, BMSC and autologous SC groups, a 100- $\mu$ l cell suspension (approximately $1 \times 10^{6}$ cells) was injected into the lesion site. For the untreated injured group, $100 \mu \mathrm{l}$ of saline was used as a control. For the autologous nerve group, the severed $1.5-\mathrm{cm}$ long sciatic nerve was flipped $180^{\circ}$ in situ and sutured using the same method. In the sham group, the skin was cut, muscles were isolated, and the sciatic nerve was exposed but not severed. The animals were housed in a standard environment. All surgical procedures minimized the number of rats used and their suffering.

Table 1. Heading?

\begin{tabular}{ll}
\hline $\begin{array}{l}\text { Groups (n=10 animals per group; unless } \\
\text { indicated otherwise) }\end{array}$ & Treatments \\
\hline Sca+B-dSC group & Scaffold+B-dSCs \\
Sca+BMSC group & Scaffold+ BMSCs \\
Sca+autologous SC group & Scaffold+autologous SCs \\
B-dSC group & B-dSC suspension \\
BMSC group & BMSC suspension \\
Autologous SC group & autologous SC suspension \\
Sca group & Scaffold \\
Control group & Autologous nerve \\
Sham group ( $=4$ animals) & Just exposed the sciatic nerve \\
Untreated group ( $\mathrm{n}=4$ animals) & Saline \\
\hline
\end{tabular}

\section{Nerve function test}

Four weeks after surgery, all groups of animals were subjected to a sciatic nerve electrophysiological examination using the RM6240 multichannel electrophysiological signal acquisition and analysis system. Briefly, the rats were anesthetized via an intraperitoneal injection of phenobarbital sodium (45 $\mathrm{mg} / \mathrm{ml}$ ). The surgical field was reopened to expose and dissect the repaired sciatic nerve. The examination of the function of the motor nerves was conducted using the procedure described below. The recording electrode was placed in the triceps surae, and the stimulating electrode was placed $0.5 \mathrm{~cm}$ from the proximal end of the sciatic nerve graft. The stimulation current, time, and frequency parameters were $1-2 \mathrm{~mA}, 0.1 \mathrm{~ms}$, and $1.0 \mathrm{~Hz}$, respectively. The amplitude (AP), latency (LT), and conduction velocity $(\mathrm{CV})$ of the nerve impulse potential were recorded.

\section{Evaluation of remyelination}

At the 4th week postoperation, the transplanted grafts and the corresponding distal nerves located 2 $\mathrm{cm}$ from the graft were isolated from all animals to be tested. The surface fat and basal lamina tissue were removed to perform the experiments listed below.

\section{TEM and toluidine blue staining}

Specimens were washed with PBS three times (5 $\mathrm{min} / \mathrm{wash})$ and fixed with a $2.5 \% \quad(\mathrm{~m} / \mathrm{v})$ glutaraldehyde solution for $2 \mathrm{~h}$, followed by $1 \%$ osmic acid for $90 \mathrm{~min}$. After dehydration in a gradient of alcohol solutions, the specimens were placed in $1 \%$ uranyl acetate overnight. Specimens were embedded in Poly/Bed 812 resin (Ted Pella, CA, USA) at $60^{\circ} \mathrm{C}$ for $48 \mathrm{~h}$ and then sectioned according to the different 
experimental designs (i.e., 0.5 - $\mu$ m-thick tissue sections were stained with toluidine blue, and the myelin sheath was observed under a light microscope (Eclipse 80i, Nikon). For TEM, ultrathin sections (80-100 nm) were prepared and stained with lead citrate and uranyl acetate. Sections were observed under a transmission electron microscope (FEI TECNAI SPIRIT G²). Previous research methods were used as references for the analysis of relevant data. Three tissue sections per group were randomly imaged using a Nikon Eclipse 80i microscope equipped with a high-resolution color digital camera (Digital Sight US-U2, Nikon). The results were analyzed using the Image-Pro Plus software (Media Cybernetics, Inc.). The following methods were used to quantify the results of the cell tracing analysis: 10 high-power fields were randomly selected, and the software was used to calculate the numbers of DAPI+ cells, EdU+ cells, and EdU+MBP+ cells. The following method was used to perform a semiquantitative analysis of the levels of the NF-200 and S100 proteins: ten high-power fields were randomly selected, and the expression levels were calculated by dividing the positively stained area for each protein by the area of the total field of view.

\section{Statistical analysis}

All experiments were repeated at least three times, unless indicated otherwise. Data are presented as means $\pm S D$ and were analyzed using the Statistical Program for Social Science (SPSS) software version 19.0. Statistical significance was estimated using one-way analysis of variance (ANOVA) with the Tukey-Kramer post hoc analysis. $\mathrm{P}$ values $<0.05$ were considered statistically significant.

\section{Results}

\section{Analysis of the scaffold properties}

In terms of structure, after acellular extraction, the nerve tissue structure was basically complete, the nuclei had been completely removed (Figure 1A), the basal lamina scaffold wall was complete, and no significant collapse of the lumen was observed (Figure 1C). The tube wall was approximately $0.5-1-\mu \mathrm{m}$-thick, and the tube diameter was generally $4-10 \mu \mathrm{m}$. The SCs formed a laminar myelin sheath structure, and the axon structure in the basal lamina scaffold had completely disappeared (Figure 1D). The interior of the scaffold showed a wavy mesh-like structure; these tubular structures guide and limit the direction of axon extension.

In terms of composition, $\mathrm{FN}$ and $\mathrm{LN}$ immunostaining (Figure 1B-C) showed that the scaffold itself contained abundant FN and LN. These protein components play important roles in seed cell adhesion and nerve regeneration.

\section{Cell culture and identification}

\section{Culture and identification of bone marrow mesenchymal cells}

After the primary cells were isolated and purified, P3 cells were collected. After $24 \mathrm{~h}$ of adherent culture, all cells showed a spindle shape under the light microscope. After 5 additional days of culture, the cells showed whirlpool-like morphology (Figure 2A). Similar to previous studies[23], flow cytometry revealed that greater than $98 \%$ of the cells expressed the mesenchymal lineage markers CD29, CD44, and CD90, and no more than 1\% of the cells expressed the hematopoietic lineage markers CD34, CD11b, CD45, and CD106 (Figure 2).

Induction, differentiation, and identification of BMSCs

The induced and differentiated B-dSCs showed an SC-like morphology and extended processes within 5-7 days (Figure 3B). According to the immunofluorescence staining, $91.3 \pm 5.4 \%(n=6)$ of cells were positive for S-100, O4, and GFAP during this period (Figure 3D-F).
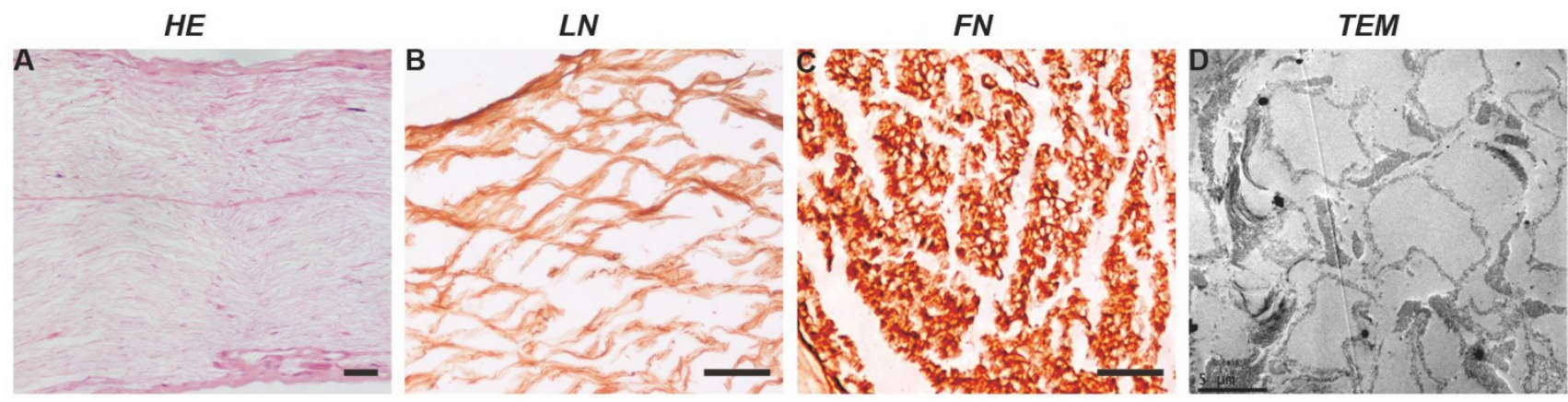

Figure 1. Basic properties of a nerve scaffold. H\&E staining shows that the nuclei in the scaffold have been completely removed. The composition test showed that the scaffold was enriched in LN (cross-section) and FN (longitudinal section). TEM image showing that the myelin sheath and axon components disappeared and that the neural basal lamina scaffold remained intact, with no significant collapse of the scaffold wall. Scale bars $=100 \mu \mathrm{m}$ in A, B and C. 

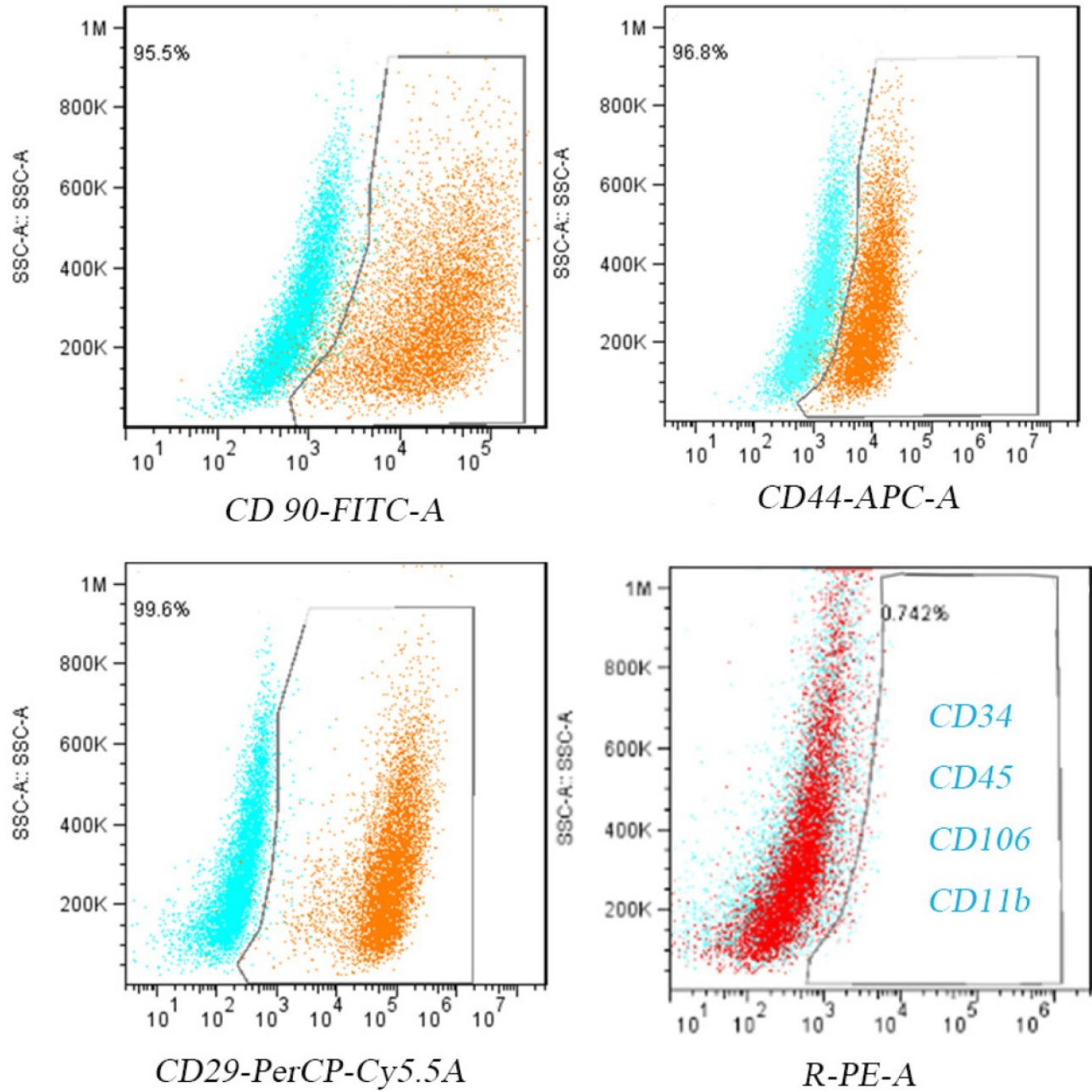

Figure 2. BMSC identification. Cultured P3 BMSCs express high levels of CD90, CD44, and CD29 and low levels of CD34,CD45,CD106 and CD11b.
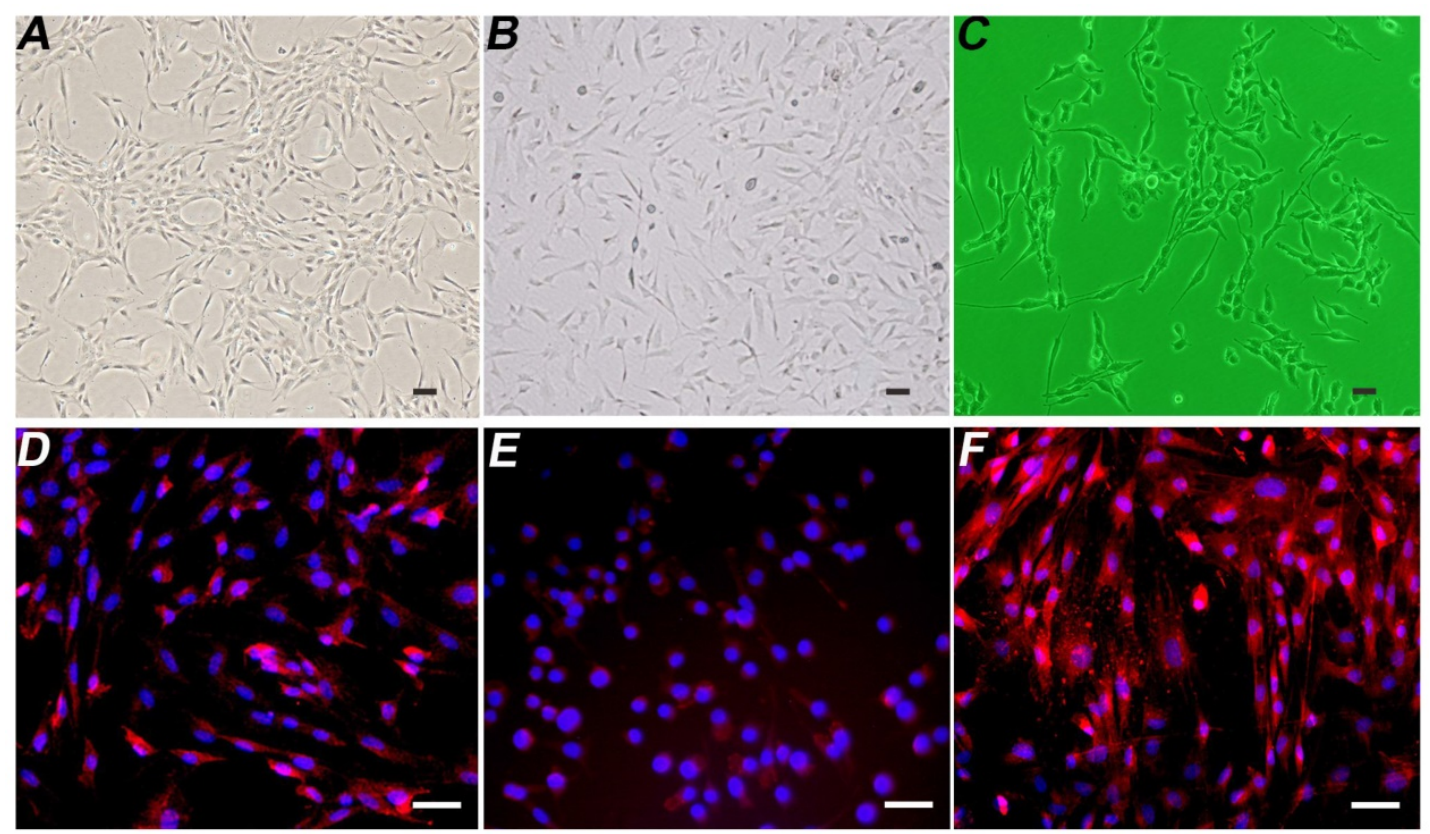

Figure 3. Detection of the properties of B-dSCs. Compared with normal BMSCs (A), the image in B shows induced B-dSCs with a disordered cell arrangement. The cell body is spindle-shaped with slender processes and has a morphology similar to SCs (C). Most of the B-dSCs expressed the SC cell-specific markers S-100 (D), O4 (E), and GFAP (F). Scale bar $=50 \mu \mathrm{m}$. 

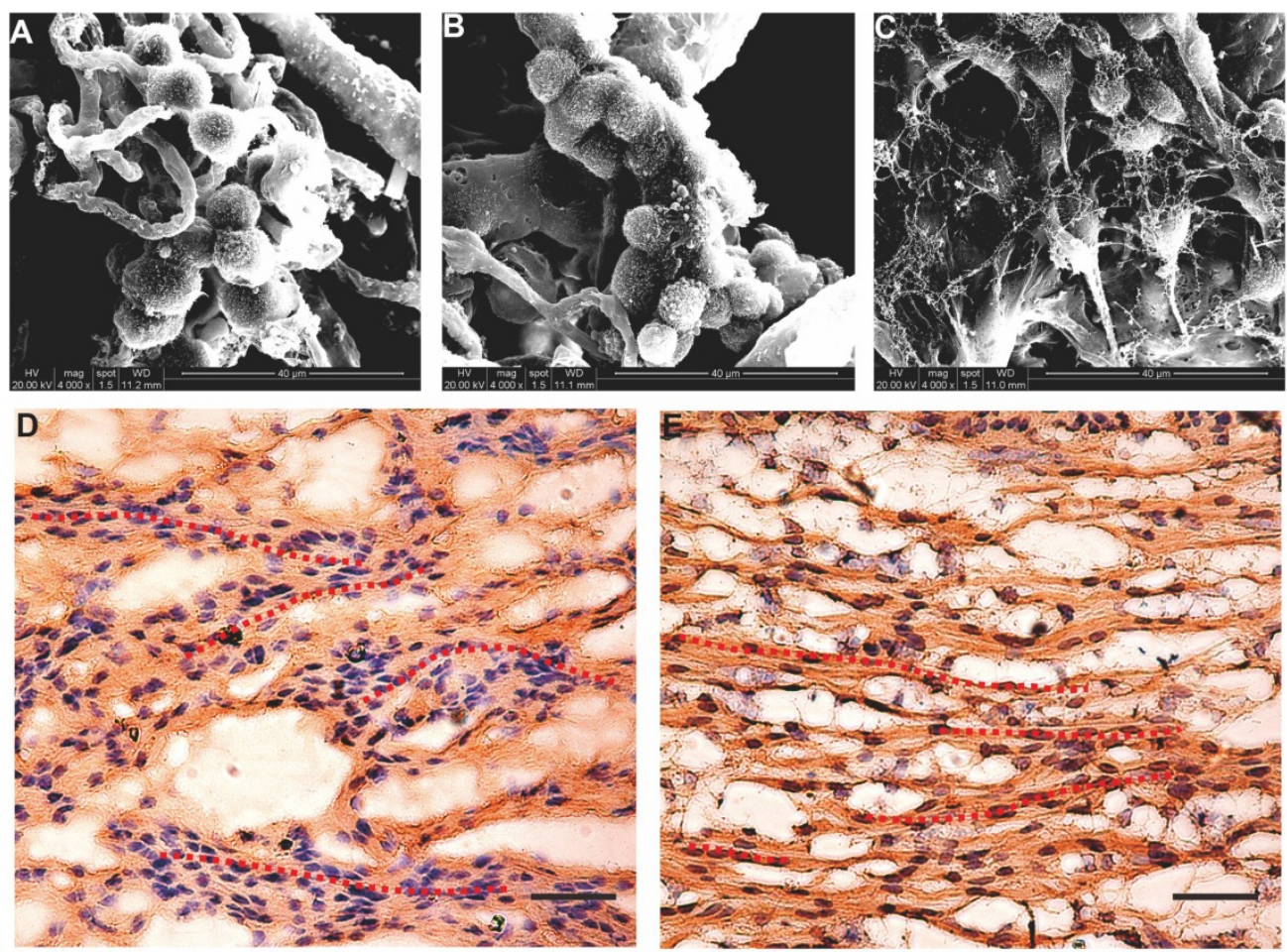

Figure 4. Composite scaffold. The cells and the scaffold were cocultured in vitro for $48 \mathrm{~h}$. A-C SEM images showing that all seed cells (A for B-dSCs; B for BMSCs; and $\mathrm{C}$ for SCs) adhere to and survive on the scaffold surface. After 7 days of coculture, immunostaining (D and E) shows stable expression of S-100 of B-dSCs and SCs cultured on the scaffold. The nuclear counterstain shows that these two cell types show a "Büngner band"-like linear arrangement on the scaffold. Scale bar=50 $\mu \mathrm{m}$.
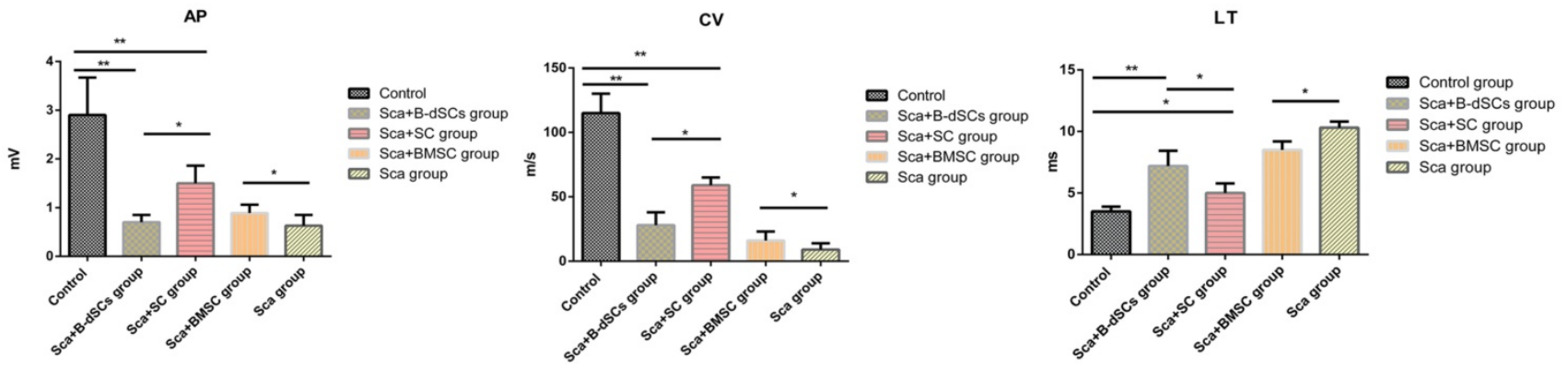

Figure 5. Electrophysiological test results. Comparison of the electrophysiological test results between groups of animals that received the scaffold transplant at four weeks after surgery. $* \mathrm{P}<0.05$, $* * \mathrm{P}<0.01$, one-way ANOVA with Tukey's post hoc test.

\section{SC culture}

The isolated and cultured autologous SCs exhibited a typical spindle-shape structure (Figure 3C).

\section{Cell labeling}

After coculture with EdU for $24 \mathrm{~h}$, the numbers of EdU+ cells among the three cell types (BMSCs, autologous SCs, and B-dSCs) were $94.5 \%, 93.9 \%$, and $97.6 \%$, respectively (Figure 6A-C).

\section{Cell complex formation}

After the seed cells were complexed with the nerve scaffold for 7 days, SEM images of the three groups of specimens showed that most of the cells aggregated and adhered to the surface of the internal pore walls of the scaffold (Figure 4A-C). Based on the immunostaining results, cells in the B-dSC and the autologous SC groups continued to express S-100 (Figure 4E-F). Thus, the acellular scaffold had better cell compatibility and represented a potentially suitable cell delivery system for the three types of cells to repair the sciatic nerve defect. Notably, these two groups of cells showed a linear arrangement under the guidance of the physical scaffold structure (see the red dotted lines in Figure 4E-F).

\section{Restoration of nerve function}

With the exception of the sham group, the posterior limbs of all animals in other groups became completely paralyzed after the nerve was severed, and motor function was completely lost. During the 
4-week observation period after surgery, all animals developed ulcers or lost toes, whereas these phenomena did not occur in the control group and the sham group. Instead, only the loss of a toenail and foot pad tissue atrophy were observed in the control group.

\section{Electrophysiological test results}

The myelin sheath plays a role in insulation and improving the nerve conduction velocity. In the electrophysiological tests, $\mathrm{CV}$ and CMAP are effective parameters that reflect the function of the myelin sheath. The results from the electrophysiological tests of the groups at four weeks after the surgery $(n=3)$ are provided below, except for the groups treated with cells alone (including BMSC, B-dSC, and autologous SC groups) and the untreated group, from which we were not able to record electrical signals because nerve regeneration did not occur in the lesion site. And atrophy of the distal nerve was observed. Rats in the Sca+B-dSC, Sca+BMSC, Sca+autologous SC and Sca groups all displayed worse recoveries than rats in the control group (CV: $115 \pm 15 \mathrm{~m} / \mathrm{s}, \mathrm{P}<0.05$; LT: $3.3 \pm 0.4 \mathrm{~ms}, \quad \mathrm{P}<0.05$; AP: $2.91 \pm 0.85 \mathrm{mV}, \quad \mathrm{P}<0.05)$.
However, the rats in the Sca+B-dSC group (CV: $28 \pm 10$ $\mathrm{m} / \mathrm{s}$; LT: $7.2 \pm 1.3 \mathrm{~ms}$; AP: $0.69 \pm 0.22 \mathrm{mV}$ ) displayed an even lower CV and CMAP than the rats in the Sca+ autologous SC group (CV: $59 \pm 6 \mathrm{~m} / \mathrm{s}$; LT: $5.1 \pm 0.8 \mathrm{~ms}$, $\mathrm{P}<0.05$; AP: $1.51 \pm 0.47 \mathrm{mV}, \mathrm{P}<0.01)$, but greater values than the rats in the Sca+BMSC group (CV: $16 \pm 3 \mathrm{~m} / \mathrm{s}$, $\mathrm{P}<0.05$; LT: $8.5 \pm 0.7 \mathrm{~ms}$; AP: $0.98 \pm 0.21 \mathrm{mV}, \mathrm{P}<0.01)$ and Sca group (CV: $9 \pm 5 \mathrm{~m} / \mathrm{s}, \mathrm{P}<0.05$; LT: $10.3 \pm 0.5 \mathrm{~ms}$; AP: $0.63 \pm 0.22 \mathrm{mV}, \mathrm{P}<0.01)$.

\section{Cell tracing and myelin-associated protein detection}

On the 7 th postoperative day $(n=4)$, none of the transplanted cells in groups treated with cells alone (BMSC, B-dSC, and autologous SC groups) had survived (data not shown). Only $7 \%$ of the transplanted cells survived in the Sca+autologous SC group, which was less than the survival rate obtained in the Sca+B-dSC (12\%) and Sca+BMSC groups (14\%). However, most (85\%) of these cells $(\mathrm{Edu}+)$ expressed $\mathrm{MBP}$, suggesting that most of these cells were capable of forming a myelin sheath in vivo. For the Sca+B-dSC and Sca+BMSC groups, MBP+EdU+ double-positive
Sca+SC group
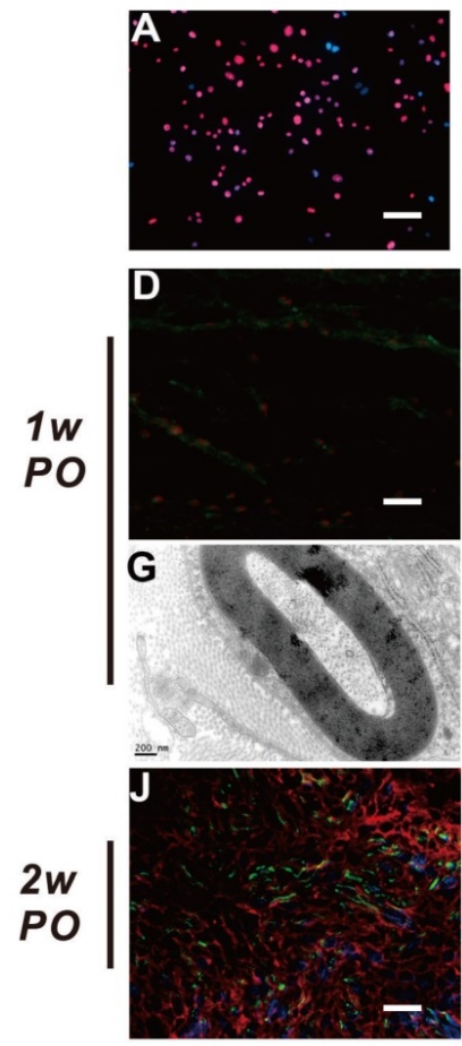

Sca+BMSC group
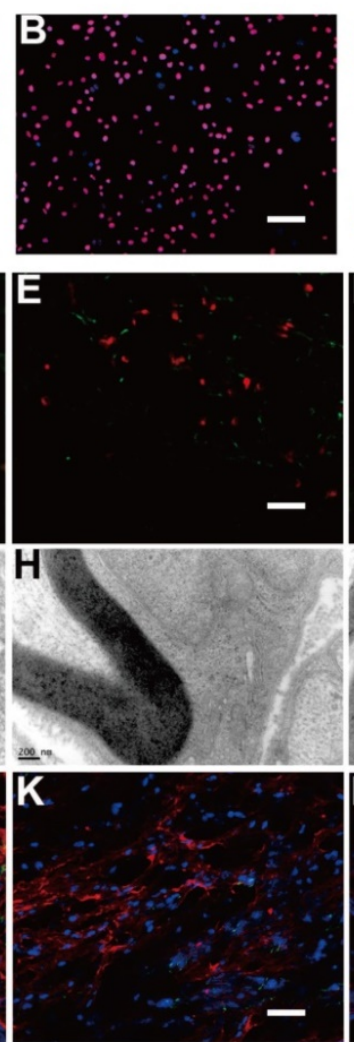

\section{Sca+B-dSCs group}
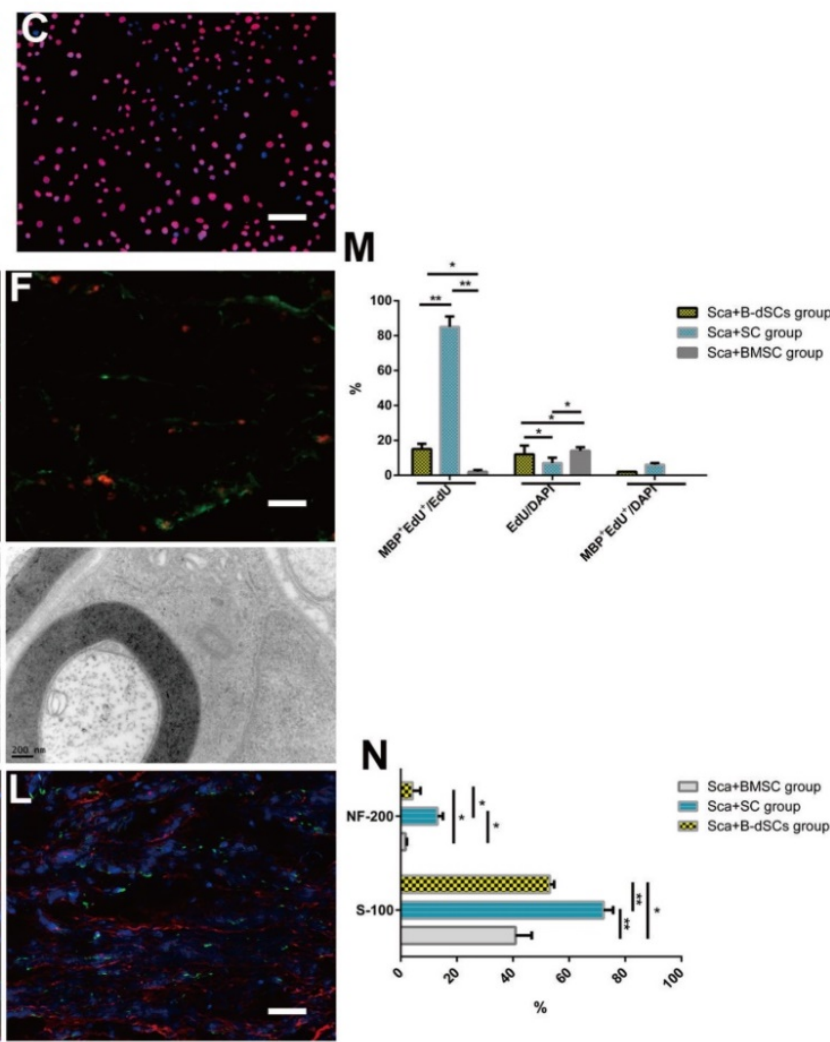

Figure 6. The role of transplanted cells in the remyelination process. A-C Images of EdU-labeled cells cultured in vitro for transplantation. D-F In the first postoperative week, cell tracing reveals that the transplanted cells (red) in each group located at the midpoint of the graft express MBP (green). The quantification of the percentages of surviving EdU+ seed cells and cells expressing MBP is shown. G-I In the first postoperative week, TEM shows that the seed cells of the three groups form myelin sheath lamellae-like ultrastructures in the scaffold. J-L Comparison of the expression of S-100 (red) and NF-200 (green) at the midpoint of the graft in the three groups during the second postoperative week. The quantitative analysis of the expression levels in the three groups is shown. $* \mathrm{P}<0.05$, $* * \mathrm{P}<0.01$, one-way ANOVA with Tukey's post hoc test. Scale bar $=50 \mu \mathrm{m}$. 
cells accounted for only $15 \%$ and $2 \%$ of the total cells, respectively, values that were significantly lower than the percentage obtained for the Sca+autologous SC group $(\mathrm{P}<0.01)$ (Figure $6 \mathrm{D}-\mathrm{F}$ and $\mathrm{M}$ ). On the 14th postoperative day, the EdU signal was not detected in these three groups of animals that received transplanted cells (data not shown). However, the quantitative results from the S-100 and NF-200 immunofluorescence staining indicated higher levels of expression of these two proteins in the Sca+autologous SC group than in the Sca+B-dSC and Sca+BMSC groups (Figure 6J-L and N), indicating better regeneration of axons and the myelin sheath in the Sca+autologous SC group than in the other two groups at this time point $(\mathrm{P}<0.05)$.

\section{Remyelination}

The proportion of myelinated nerves and the depth and depth distribution of the myelin sheaths are powerful indicators of remyelination. During the 4 th postoperative week $(n=4)$, lesion sites were reexposed, and groups transplanted with cells alone (BMSC, B-dSC, and autologous SC groups) and the untreated group did not display obvious nerve regeneration in the defect area, defect areas exceeding $1.5 \mathrm{~cm}$ in length were observed between the stumps of the nerve, and the distal nerve tissue exhibited obvious shrinkage. Therefore, the remyelination evaluation was mainly aimed at the groups in which the scaffold was transplanted (Sca+B-dSC, Sca+ BMSC, Sca+autologous SC, and Sca groups), as well as the control group.

The toluidine blue and TEM data (Figure 7) indicated that $45 \%$ of the nerve fibers in the grafts of the Sca+B-dSC group were myelinated, a value that was significantly lower than the proportion in the Sca+autologous SC group $(70 \%$; $<<0.01)$, did not differ from the proportion of the Sca+BMSC group (43\%) $(P>0.05)$ and was slightly higher than the proportion of the Sca group $(31 \%)(P>0.05)$. However, the values obtained for these four groups were less than the values obtained for the control group $(P>0.05)$. The average thickness of the myelin sheath in the Sca+ autologous SC group was $0.73 \pm 0.19 \mu \mathrm{m}$, which was significantly higher than the thickness of $0.42 \pm 0.05 \mu \mathrm{m}$ observed in the Sca+B-dSC group, $0.19 \pm 0.07 \mu \mathrm{m}$ in the Sca+BMSC group and $0.21 \pm 0.02 \mu \mathrm{m}$ in the Sca group. The G-ratio was $0.64 \pm 0.04$ in the control group but only $0.88 \pm 0.06$ in the Sca+B-dSC group, which was higher than the $0.73 \pm 0.09$ ratio $(\mathrm{P}<0.05)$ obtained for the Sca+autologous SC group. No significant difference was detected compared to the $0.91 \pm 0.03$ ratio $(\mathrm{P}>0.05)$ obtained for the Sca+BMSC group and $0.87 \pm$ 0.09 in the Sca group $(\mathrm{P}>0.05)$. Based on the analysis of the thickness of the myelin sheath, the distribution of myelin sheaths with a thickness greater than $0.6 \mu \mathrm{m}$ was as follows: graft $74 \%$ and distal nerve $20 \%$ in the Sca+autologous SC group; graft 20\% and distal nerve $5 \%$ in the Sca+B-dSC group; graft $10 \%$ and distal nerve $2 \%$ in the Sca+BMSC group; graft $71 \%$ and distal nerve $29 \%$ in the Sca group and graft $80 \%$ and distal nerve $73 \%$ in the control group. The toluidine blue and TEM results showed that the numbers of axons in the central region of the graft and in the distal nerves in the animals of the Sca+B-dSC, Sca+ autologous SC, and Sca+BMSC groups were $487 \pm 89$ and $276 \pm 66,560 \pm 41$ and $327 \pm 37$, and $521 \pm 33$ and $232 \pm 59$, respectively. No significant differences were observed among the three groups. However, the values of the Sca+BMSC groups were significantly higher than the Sca group $(256 \pm 19$ and $188 \pm 35$, respectively; $\mathrm{P}<0.05)$.

\section{Discussion}

A nerve scaffold is a necessary prerequisite for the repair of defective nerves. If no bridging scaffold is available, the ability of regenerated axons to cross the injured area is hindered for long-distance nerve defects[24]. The nerve scaffold provides a good microenvironment for nerve regeneration, in which SCs adhere to and migrate along the scaffold surface [25]. The spatial structure of the scaffold provides natural physical guidance for the SCs, enabling them to eventually form a unique cell alignment structure (Büngner band), which is the most important condition for inducing axonal extension[26]. After the cells were seeded on the surface of the scaffold and cocultured for 7 days, the cells (B-dSCs and autologous SCs in our experiment) showed an ordered cell alignment under the guidance of the natural scaffold structure (see Figure 4D-E). We postulate that this cell structure plays an important role in the subsequent regeneration of nerve axons. A simple acellular nerve scaffold has been reported to promote a certain degree of myelin regeneration (see our data in the results section), but this approach still has many problems compared with SCs embedded in a scaffold [27]. Therefore, supplementation with exogenous SCs is necessary. The scaffold itself provides only a substrate for the adhesion and migration of cells, and when the number of SCs is insufficient to assist in the regeneration of the nerve axons, the efficacy of nerve scaffold is reduced. Simple transplantation of cells may promote regeneration under conditions in which the continuity of nerve is intact; however, for long-distance nerve defects, locally injected cells did not increase regeneration in the absence of a scaffold (on the 7th day after the operation, locally injected cells were unable to survive without a scaffold). As expected, in this experiment, the isolated cell transplantation groups and the 
TEM

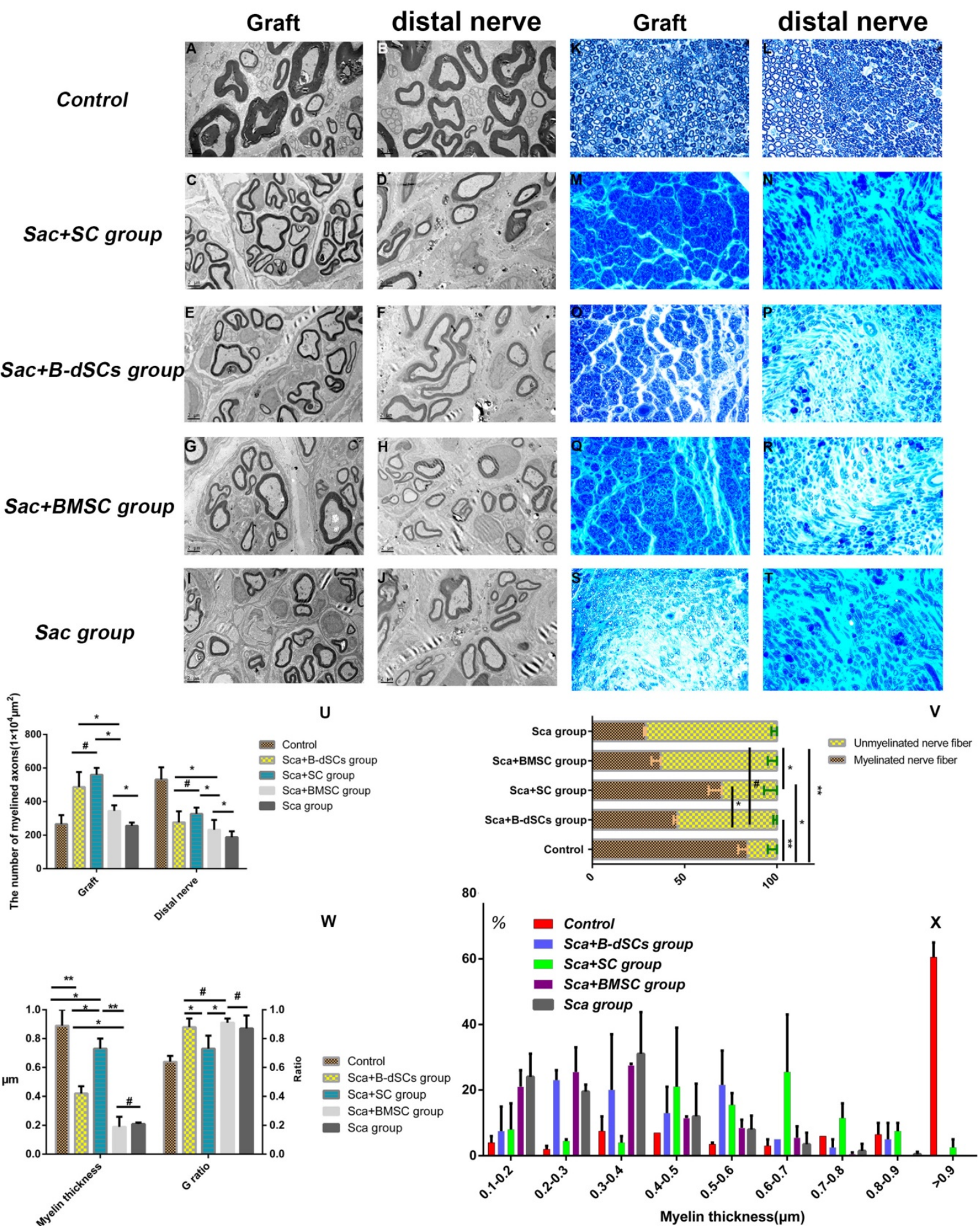

Figure 7. Comparison of remyelination between groups. At 4 weeks after surgery, the midpoint of the graft and the distal nerve were examined using toluidine blue staining $(\mathrm{K}-\mathrm{T} ; 400 \times)$ and TEM $(\mathrm{A}-\mathrm{J} ; \mathrm{bar}=2 \mu \mathrm{m})$. The results of statistical analyses comparing the number of regenerated myelinated/unmyelinated nerves $(U)$, their ratios $(V)$, the thickness of the regenerated myelin sheath, and the G-ratio $(W)$ are shown. $(X)$ Detailed distribution of the mean myelin sheath thickness in the midpoint of the graft and the distal nerve of the four groups. $* \mathrm{P}<0.05, * * \mathrm{P}<0.01, \# \mathrm{P}>0.05$, one-way ANOVA with Tukey's post hoc test. Scale bar $=200 \mu \mathrm{m}$.

untreated injury group did not reconstruct the continuous nerve by the 4th week after surgery. Therefore, the role of the scaffold is very important.
We postulate that scaffold implantation is a prerequisite for the evaluation of exogenous cellular function in long-distance nerve defect models. 
The regeneration and extension of axons are inseparable from the effects of SCs[28-31]. When the nerves are severed, the injured area lacks a sufficient number of SCs to support axon regeneration. Strategies that rely solely on the entry of proliferating SCs at the proximal stump into the scaffold and the subsequent induction of nerve regeneration is obviously unrealistic. Thus, exogenous SCs are an important tool to assist in nerve regeneration[4]. BMSCs have the ability to differentiate into ectodermal neuronal cells[32]. Monolayers of B-dSCs have recently been shown express their specific markers (S-100, O4, and GFAP)[33], and differentiated cells have the ability to perform the basic functions of SCs, such as participating in the ATP-induced intracellular Ca2+ signaling pathway (ATP-purinergic signaling) [34], the secretion of multiple neurotrophic factors [34], inducing the extension of neuronal axons, and wrapping new axons with myelin sheaths[18]. These characteristics confirm that B-dSCs are more effective than BMSCs at promoting nerve regeneration and are ideal substitutes for SCs[36,37]. In this experiment, we used the scaffold to simulate the 3-D environment in vivo and found that both B-dSCs and SCs continued to express the S-100 protein when the seed cells were complexed with the scaffold for 7 days. Based on this finding, the physical environment created by the scaffold does not change the basic characteristics of B-dSCs. Thus, the scaffold can be used as a vehicle for in vivo cell delivery. However, the role of SCs is extremely complex in the in vivo environment. Ideal seed cells should perform the same functions as genuine SCs. What are the actual functions of B-dSCs in vivo?

We conducted in vivo experiments to answer these questions. In the present study, animals in the autologous nerve transplantation group showed only mild foot pad and toenail atrophy during the 4-week observation period. During the first four weeks after the operation, all animals in the other groups (except for the sham group) showed different proportions of ulcers or toe loss. Neurotrophic substances are synthesized by the soma of neurons and are transported through axoplasm flow to the nerve endings for release[38]. This process does not affect the conduction of nerve impulses. Even if the conduction of nerve impulses is abnormal (e.g., due to the myelin sheath), the axoplasm flow will not stop, and the tissue under its innervation will not undergo metabolic changes and atrophy[39]. Therefore, we preliminarily suggested that the nerve repair in the Sca+B-dSC, Sca+autologous SC, and Sca+BMSC groups did not reach the same efficacy as autologous nerve transplantation. We subsequently performed electrophysiological examinations to further explore the evidence of remyelination and repair in the Sca+B-dSC, Sca+autologous SC, and Sca+BMSC groups. In the electrophysiological analysis, the $\mathrm{CV}$, LT, and AMP were significantly different in the Sca+B-dSC or Sca+BMSC group compared with the Sca+autologous SC group. SCs are the cell type responsible for the formation of myelin, and the quality of the resulting myelin sheath (i.e., the number of myelinated nerve fibers, the thickness of the myelin sheath, and the distance between the nodes of Ranvier) is a critically important factor that affects the electrophysiological outcome[40,41]. Therefore, we speculate that B-dSCs may not be able to perform all functions of autologous SCs, particularly the formation of the myelin sheath, and thus, a difference may exist between the two cell types.

We analyzed the differences in the promotion of myelin sheath formation by the seed cells in each group using cell tracing and morphology to verify this hypothesis. The thickness of the myelin sheath, the proportion of myelinated fibers, and the myelin area ratio were reduced in the Sca+B-dSC group compared with the Sca+autologous SC group. In particular, regarding the distribution of the myelin sheath, approximately $70 \%$ of myelin sheaths had a thickness $>0.6 \mu \mathrm{m}$ in the Sca+autologous SC group, whereas this value was less than $25 \%$ in the Sca+B-dSC group. We investigated whether these results were caused by differences in the survival rates of the seed cells. We tried to analyze the results using the cell tracing technique to exclude this possibility. On the 7th postoperative day, the tracing results showed that more transplanted BMSCs and B-dSCs survived than autologous SCs. However, based on the ratio of $\mathrm{MBP}+\mathrm{EdU}+$ cells, a significantly greater proportion of cells formed myelin sheaths in the Sca+autologous SC group than in the Sca+B-dSC group. These data most likely confirmed that a large difference existed in the abilities of B-dSCs and autologous SCs to form the myelin sheath. Most B-dSCs do no function like SCs in vivo, as expected. We speculated that a possible explanation was that some of the B-dSCs failed to be converted to the myelinating states that effectively wrap the axon and form the myelin sheath, thus increasing the number of unmyelinated fibers[42]. The nonmyelinating states are important conditions for maintaining the integrity of myelin[43]. According to the electron microscopy results, the myelin sheath structures formed by the B-dSCs, autologous SCs, and BMSCs were intact. We postulate that some B-dSCs are converted to nonmyelinating states in vivo, but in-depth in vivo studies are needed to clarify this speculation. The survival and maintenance of related Schwann cell properties in the repair state are the keys to successful nerve regeneration[5]. The so-called 
Büngner band structure is actually formed by Schwann cells in the repair state[44]. Both the Sca+B-dSC and the Sca+autologous SC groups formed cell alignments under the guidance of the physical structure of the scaffolds and formed a Büngner band-like structure to induce axon regeneration and extension[6]. We hypothesized that the seed cells of the Sca+B-dSC and Sca+autologous SC groups served to stabilize the Schwann cells in the repair state. The use of acellular nerve allografts (ANGs) combined with B-dSCs or BMSCs results in less axon regeneration than ANGs containing SCs[34,45]. In the present study, the analysis of the axon regeneration revealed lower levels of NF-200 and S-100 immunostaining in the Sca+B-dSC group during the second postoperative $\mathrm{w}$ than in the Sca+autologous SC group, although the expression levels were higher than those in the Sca+BMSC group. However, 4 weeks after the operation, the analysis of the number of regenerated axons showed significant differences among the Sca+B-dSC, Sca+autologous SC, and Sca+BMSC groups. Based on this result, we speculated that autologous SCs formed a Büngner band on the scaffold in the early stage after the operation and were induced axon extension to a greater extent than the other two cell types. Regarding the results obtained in the later stage, one possible explanation is that B-dSCs or BMSCs may promote nerve regeneration through other pathways[46-48], such as the secretion of certain neurotrophic factors, local regulation of immunity, or the induction of angiogenesis, and the comprehensive effects of these alternative pathways may partially compensate for some of the defects of B-dSCs or BMSCs in promoting remyelination. Based on the above analysis, we postulate that B-dSCs may play a certain role in the myelination of the severed nerves but do not completely replace autologous SCs. However, the relevant mechanisms require further exploration.

\section{Conclusions}

B-dSCs are a type of commonly used seed cell for nerve repair. When combined with an acellular nerve scaffold, they promote nerve regeneration to a certain extent. However, significant differences were observed compared with autologous SCs in vivo. In the first 4 postoperative weeks, B-dSCs do not achieve the same effect on myelination as autologous SCs but are better than BMSCs. The underlying mechanism requires further exploration.

\section{Acknowledgements}

This work was supported by grants from the Science and Technology Program of Guangzhou, China (201604020080) and the Science and Technology
Project of Guangdong Province (2015A020212016 and 2016A020214007).

\section{Competing Interests}

The authors have declared that no competing interest exists.

\section{References}

1. Armstrong S J, Wiberg M, Terenghi G, et al. ECM molecules mediate both Schwann cell proliferation and activation to enhance neurite outgrowth. Tissue Eng. 2007; 13(12): 2863-2870.

2. Campana W M. Schwann cells: activated peripheral glia and their role in neuropathic pain. Brain Behav Immun. 2007; 21(5): 522-527.

3. Ide C. Peripheral nerve regeneration. Neurosci Res. 1996, 25(2): 101-121.

4. Kim H A, Mindos T, Parkinson D B. Plastic fantastic: Schwann cells and repair of the peripheral nervous system. Stem Cells Transl Med. 2013; 2(8): 553-557.

5. Jessen K R, Mirsky R. The repair Schwann cell and its function in regenerating nerves. J Physiol. 2016; 594(13): 3521-3531.

6. Jessen K R, Mirsky R, Lloyd A C. Schwann Cells: Development and Role in Nerve Repair. Cold Spring Harb Perspect Biol. 2015; 7(7): a20487.

7. Allodi I, Udina E, Navarro X. Specificity of peripheral nerve regeneration: interactions at the axon level. Prog Neurobiol. 2012; 98(1): 16-37.

8. Webber C, Zochodne D. The nerve regenerative microenvironment: early behavior and partnership of axons and Schwann cells. Exp Neurol. 2010;223(1): 51-59.

9. Grim M, Halata Z, Franz T. Schwann cells are not required for guidance of motor nerves in the hindlimb in Splotch mutant mouse embryos. Anat Embryol (Berl). 1992; 186(4): 311-318.

10. Deumens R, Bozkurt A, Meek M F, et al. Repairing injured peripheral nerves: Bridging the gap. Prog Neurobiol. 2010, 92(3): 245-276.

11. Dubovy P. Wallerian degeneration and peripheral nerve conditions for both axonal regeneration and neuropathic pain induction. Ann Anat. 2011; 193(4): 267-275.

12. Navarro X, Vivo M, Valero-Cabre A. Neural plasticity after peripheral nerve injury and regeneration. Prog Neurobiol. 2007; 82(4): 163-201.

13. Daly W, Yao L, Zeugolis D, et al. A biomaterials approach to peripheral nerve regeneration: bridging the peripheral nerve gap and enhancing functional recovery. J R Soc Interface. 2012;9(67): 202-221.

14. Fansa H, Dodic $T$, Wolf $G$, et al. Tissue engineering of peripheral nerves: Epineurial grafts with application of cultured Schwann cells. Microsurgery. 2003; 23(1): 72-77.

15. Qiu X C, Jin H, Zhang R Y, et al. Donor mesenchymal stem cell-derived neural-like cells transdifferentiate into myelin-forming cells and promote axon regeneration in rat spinal cord transection. Stem Cell Res Ther. 2015; 6: 105.

16. Dezawa M, Takahashi I, Esaki M, et al. Sciatic nerve regeneration in rats induced by transplantation of in vitro differentiated bone-marrow stromal cells. Eur J Neurosci. 2001;14(11): 1771-1776.

17. Wang X, Luo E, Li Y, et al. Schwann-like mesenchymal stem cells within vein graft facilitate facial nerve regeneration and remyelination. Brain Res. 2011;1383: 71-80

18. Cai S, Tsui Y P, Tam K W, et al. Directed Differentiation of Human Bone Marrow Stromal Cells to Fate-Committed Schwann Cells. Stem Cell Reports. 2017; 9(4): 1097-1108

19. Wang T, Yang C. Bone marrow stroma cells differentiate into Schwann cells for regeneration of peripheral nerve [M]. 2003; 580-581.

20. Ao Q, Fung C K, Tsui A Y, et al. The regeneration of transected sciatic nerves of adult rats using chitosan nerve conduits seeded with bone marrow stromal cell-derived Schwann cells. Biomaterials. 2011;32(3): 787-796.

21. Pan Y, Cai S. Current state of the development of mesenchymal stem cells into clinically applicable Schwann cell transplants. Mol Cell Biochem. 2012; 368(1-2): 127-135.

22. Kotogany E, Dudits D, Horvath G V, et al. A rapid and robust assay for detection of S-phase cell cycle progression in plant cells and tissues by using ethynyl deoxyuridine. Plant Methods. 2010; 6(1): 5.

23. Sun S, Guo Z, Xiao X, et al. Isolation of mouse marrow mesenchymal progenitors by a novel and reliable method. Stem Cells. 2003, 21(5): 527-535.

24. Tian L, Prabhakaran M P, Ramakrishna S. Strategies for regeneration of components of nervous system: scaffolds, cells and biomolecules. Regen Biomater. 2015; 2(1): 31-45.

25. Szynkaruk M, Kemp S W, Wood M D, et al. Experimental and clinical evidence for use of decellularized nerve allografts in peripheral nerve gap reconstruction. Tissue Eng Part B Rev. 2013; 19(1): 83-96.

26. Georgiou M, Bunting S C, Davies H A, et al. Engineered neural tissue for peripheral nerve repair. Biomaterials. 2013; 34(30): 7335-7343.

27. Sun $\mathrm{X} \mathrm{H}, \mathrm{Che} Y \mathrm{Q}$, Tong $\mathrm{X} \mathrm{J}$, et al. Improving nerve regeneration of acellular nerve allografts seeded with SCs bridging the sciatic nerve defects of rat. Cell Mol Neurobiol, 2009;29(3): 347-53

28. Suter U, Scherer S S. Disease mechanisms in inherited neuropathies. Nat Rev Neurosci. 2003;4(9): 714-726. 
29. Fu S Y, Gordon T. The cellular and molecular basis of peripheral nerve regeneration. Mol Neurobiol. 1997; 14(1-2): 67-116.

30. Zhou Y, Notterpek L. Promoting peripheral myelin repair. Exp Neurol. 2016;283(Pt B): 573-580.

31. Bunge R P. The role of the Schwann cell in trophic support and regeneration. J Neurol. 1994;242(1 Suppl 1): S19-S21.

32. Caddick J, Kingham P J, Gardiner N J, et al. Phenotypic and functional characteristics of mesenchymal stem cells differentiated along a Schwann cell lineage. Glia. 2006; 54(8): 840-849.

33. Wang $\mathrm{Y}$, Zhao Z, Ren $\mathrm{Z}$, et al. Recellularized nerve allografts with differentiated mesenchymal stem cells promote peripheral nerve regeneration. Neurosci Lett. 2012; 514(1): 96-101.

34. Rutten M J, Janes M A, Chang I R, et al. Development of a functional schwann cell phenotype from autologous porcine bone marrow mononuclear cells for nerve repair. Stem Cells Int. 2012; 2012: 738484.

35. Mahay D, Terenghi G, Shawcross S G. Schwann cell mediated trophic effects by differentiated mesenchymal stem cells. Exp Cell Res. 2008; 314(14): 2692-2701.

36. Keilhoff G, Goihl A, Langnase K, et al. Transdifferentiation of mesenchymal stem cells into Schwann cell-like myelinating cells. Eur J Cell Biol. 2006; 85(1): $11-24$.

37. Wakao S, Hayashi T, Kitada M, et al. Long-term observation of auto-cell transplantation in non-human primate reveals safety and efficiency of bone marrow stromal cell-derived Schwann cells in peripheral nerve regeneration. Exp Neurol. 2010; 223(2): 537-547.

38. Baumann N, Pham-Dinh D. Biology of oligodendrocyte and myelin in the mammalian central nervous system. Physiol Rev. 2001; 81(2): 871-927.

39. Young W. Electrical stimulation and motor recovery. Cell Transplant. 2015;24(3): 429-446.

40. Chen Z L, Yu W M, Strickland S. Peripheral regeneration. Annu Rev Neurosci. 2007:30: 209-233.

41. Nave K A, Werner H B. Myelination of the nervous system: mechanisms and functions. Annu Rev Cell Dev Biol. 2014;30: 503-533.

42. Griffin J W, Thompson W J. Biology and pathology of nonmyelinating Schwann cells. Glia. 2008;56(14): 1518-1531.

43. Jessen K R, Mirsky R. The origin and development of glial cells in peripheral nerves. Nat Rev Neurosci. 2005; 6(9): 671-682.

44. Arthur-Farraj P J, Latouche M, Wilton D K, et al. c-Jun reprograms Schwann cells of injured nerves to generate a repair cell essential for regeneration. Neuron. 2012; 75(4): 633-647.

45. Keilhoff G, Goihl A, Stang F, et al. Peripheral nerve tissue engineering: autologous Schwann cells vs. transdifferentiated mesenchymal stem cells. Tissue Eng. 2006, 12(6): 1451-1465.

46. Wakao S, Matsuse D, Dezawa M. Mesenchymal stem cells as a source of Schwann cells: their anticipated use in peripheral nerve regeneration. Cells Tissues Organs. 2014; 200(1): 31-41.

47. Oliveira J T, Mostacada K, de Lima S, et al. Bone marrow mesenchymal stem cell transplantation for improving nerve regeneration. Int Rev Neurobiol. 2013; 108: 59-77.

48. Zhang $\mathrm{Y}$, Luo H, Zhang Z, et al. A nerve graft constructed with xenogeneic acellular nerve matrix and autologous adipose-derived mesenchymal stem cells. Biomaterials. 2010; 31(20): 5312-5324. 